\title{
Epithelial-mesenchymal transition and cancer stem cell-like phenotype induced by Twist1 contribute to acquired resistance to irinotecan in colon cancer
}

\author{
YONG YANG $^{1 *}$, GUOXIN WANG $^{1,2 *}$, DAJIAN ZHU ${ }^{3 *}$, YANFENG HUANG $^{4}$, \\ YONG LUO ${ }^{1}$, PENGFEI SU ${ }^{1}$, XIAOWU CHEN ${ }^{1}$ and QIAN WANG ${ }^{2}$
}

${ }^{1}$ Department of Gastrointestinal Surgery, Shunde First People's Hospital Affiliated to Southern Medical University, Shunde, Guangdong 528300; ${ }^{2}$ Department of Hepatobiliary Surgery, The First Affiliated Hospital of Sun Yat-Sen University, Guangzhou, Guangdong 510080; ${ }^{3}$ Department of Gastrointestinal Surgery, Shunde Women and Children's Hospital Affiliated to Jinan University, Shunde, Guangdong 528300; ${ }^{4}$ Department of Traditional Chinese Medicine, Shunde

First People's Hospital Affiliated to Southern Medical University, Shunde, Guangdong 528300, P.R. China

Received December 12, 2016; Accepted April 7, 2017

DOI: $10.3892 /$ ijo.2017.4044

\begin{abstract}
Inherent and acquired chemoresistance reduce the effectiveness of irinotecan in the treatment of metastatic colorectal cancer (CRC). However, the molecular mechanisms underlying this resistance process are still unclear. Twist1 is one of the master transcription factors of epithelial-mesenchymal transition (EMT). Our previous study indicated that Twist 1 is overexpressed in colon cancer tissues, and demonstrated that Twist1 plays a crucial role in the chemoresistance of CRC. In the present study, we further investigated how Twist 1 contribute to acquired resistance to irinotecan in colon cancer. The irinotecan-resistant cells were established by gradual adaptation of increasing irinotecan concentrations in LoVo cells, named LoVo/CPT-11R cells. Results showed that cell viabilities to different anticancer drugs were markedly increased in LoVo/CPT-11R cells compared to LoVo cells. Moreover, LoVo/CPT-11R cells displayed EMT, CSC-like
\end{abstract}

Correspondence to: Professor Xiaowu Chen, Department of Gastrointestinal Surgery, Shunde First People's Hospital Affiliated to Southern Medical University, Shunde, Guangdong 528300, P.R. China

E-mail: drchenxiaowu@163.com

Professor Qian Wang, Department of Hepatobiliary Surgery, The First Affiliated Hospital of Sun Yat-sen University, Shunde, Guangzhou 510080, P.R. China

E-mail: wangqian_dr@126.com

"Contributed equally

Abbreviations: CRC, colorectal cancer; EMT, epithelialmesenchymal transition; CSC, cancer stem cell; CD, cluster of differentiation; MMP, metalloprotemase

Key words: colon cancer, irinotecan, resistance, epithelial-mesenchymal transition, cancer stem cell, Twist1 cellular morphology and relative biomarkers were also significantly increased. In addition, overexpressed Twist1 LoVo cells were established by lentivirus transfection assay, named LoVo/Twist1 cells. Results showed that the LoVo/Twist1 cells perform a distinctly decreased sensitivity to irinotecan, downregulated expression of E-cadherin, upregulated expression of cluster of differentiation 44 (CD44), and a significant enhancement of invasion and migration potential by regulation of MMP2 compared with control cells. In contrast, the inhibition of Twist1 transfected with siRNA could enhance the irinotecan sensitivity in LoVo/CPT-11R cells and downregulate the expression of vimentin and CD44. Our data provide evidence that EMT and CSC-like phenotype induced by Twist 1 contribute to acquire resistance to irinotecan and enhanced migration and invasion in colon cancer.

\section{Introduction}

Colorectal cancer (CRC), including colon and rectal cancer, is the third most commonly diagnosed malignancy and the fourth leading cause of cancer-related deaths all over the world. Rapid increases in both CRC incidence and mortality are now observed with the rapid social and economic changes in Eastern Europe, Asia and South America (1). Although improved treatment strategies involving surgery and chemoand radio-therapy have increased the overall survival rates in the early stages, $40-50 \%$ of all patients with CRC present with metastasis either at the time of diagnosis or as recurrent disease upon intended curative therapy (2). Currently, irinotecan (CPT-11) is mainly used in CRC diagnosed patients with metastases, with recorded relapse or progression after application of standard 5-FU based therapy $(3,4)$. However, the response rate to these regimens is only in the range of $\sim 30-55 \%$ and the 5-year survival rate is less than $10 \%$. Resistance to chemotherapy is a major limitation to the outcome in CRC $(5,6)$. Irinotecan, a semi-synthetic analog of camptothecin, is a broad-spectrum anticancer drug that specifically target DNA topoisomerase I (Topo I). In humans, 
irinotecan is metabolized by an endogenous carboxylesterasemediated hydrolyzation into a highly active metabolite, SN-38. The formation of SN-38-Topo I-DNA complex results in lethal double-strand DNA breakage and cell death $(7,8)$. Numerous studies have been done to uncover possible mechanisms for the cellular resistance to this agent, suggesting the following general aspects: i) variable levels of the enzymes involved in the conversion of irinotecan; ii) reduced cellular accumulation from active drug efflux caused by ABC transporters; iii) changed activity of Topo I that decreases levels of the SN-38-TopoI-DNA complex; and iv) alterations in the events downstream from the ternary complex, for example, apoptosis, cell cycle regulation, checkpoints and DNA repair $(3,7,9)$. However, research on the mechanism underlying the resistance to irinotecan is still limited and needs further investigation.

Epithelial-mesenchymal transition (EMT) is a unique process initially characterized in embryonic development in which cells lose epithelial features and gain mesenchymal properties. EMT results in epithelial cells becoming spindle shaped, with loss of cellular polarity similar to mesenchymal cells. These phenotypic changes closely correlate with increased cellular motility, invasion and therapeutic resistance $(2,10)$. Furthermore, cells with different molecular characteristics within the same tumor respond differently to anticancer therapeutics, leading to drug resistance. Cancer cells may also undergo adaptive changes following therapy, exacerbating drug resistance. In epithelial cancers, these adaptive changes may involve, at least in part, EMT and the reverse process (MET) (11). Notably, these properties have also been ascribed to normal stem cells and cancer stem cells. The cancer stem cells (CSC) are specific undifferentiated cancer cells, the tumor-initiating cells, have the ability to self-renew, propagate and differentiate leading to cancer growth and progression (12). Likewise, CSCs display aggressive characteristics including increased invasion, metastatic ability and resistance to therapy and predict poor patient prognosis. In experimental models, CSCs are more resistant than differentiated tumor cells to chemo- and radiotherapy, and they can escape from the effects of conventional cytotoxic treatments (13).

Twist is a basic helix-loop-helix transcription factor, which is one of the master regulators of EMT process, including Twist1 and Twist2 (14). Our previous study indicated that Twist1 is overexpressed in colon cancer tissue, its positive expression is related to histological grade, TNM stage, recurrence and poor overall survival and is a significant independent prognostic indicator in CRC patients (15). Different studies have also reported that high expression of Twist1 is closely associated with more aggressive behavior of breast (16), hepatocellular (17), pancreatic (18) and esophageal squamous cell cancer (19). In addition, Twist is critical for the maintenance of EMT associated CSC-like characteristics $(20,21)$. Moreover, Twist1 is also responsible for paclitaxel resistance in breast cancer cells (16), cisplatin resistance in lung cancer cells (22) and 5-FU resistance in colon cancer cells (23). However, the relationship Twist1, CSC and resistance to irinotecan in colon cancer is still ambiguous. A better understanding of these underlying resistance mechanisms is a major concern for the future development of new Topo I inhibitors and the identification of biomarkers that could be used to predict tumor response to these drugs clinically.

\section{Materials and methods}

Cell culture and establishment of irinotecan-resistant LoVo cell subline. Colon cancer LoVo cells were purchased from the Type Culture Collection of the Chinese Academy of Sciences (Shanghai, China). The LoVo cells were cultured as monolayers in RPMI-1640 medium (Gibco, Carlsbad, CA, USA) supplemented with $10 \%$ fetal bovine serum (FBS; Gibco, São Paulo, Brazil) and 1\% penicillin/streptomycin (Beijing Solarbio Science and Technology, Co. Ltd., China). Cells were grown at $37^{\circ} \mathrm{C}$ in a humidified atmosphere containing $5 \% \mathrm{CO}_{2}$. Irinotecan-resistant LoVo cell subline, referred to as LoVo/CPT-11R cells, were established by gradual adaptation of the original cell line to increasing irinotecan concentrations over a period of 10 months. The concentrations increased as follows: $1 \mu \mathrm{g} / \mathrm{ml} \rightarrow 3 \mu \mathrm{g} / \mathrm{ml} \rightarrow 5 \mu \mathrm{g} / \mathrm{ml} \rightarrow 10 \mu \mathrm{g} / \mathrm{ml} \rightarrow 20 \mu \mathrm{g} / \mathrm{ml}$ $\rightarrow 30 \mu \mathrm{g} / \mathrm{ml} \rightarrow 40 \mu \mathrm{g} / \mathrm{ml} \rightarrow 50 \mu \mathrm{g} / \mathrm{ml} \rightarrow 60 \mu \mathrm{g} / \mathrm{ml} \rightarrow 70 \mu \mathrm{g} / \mathrm{ml}$. Cells were maintained at a particular irinotecan concentration for $\sim 10$ passages or until they displayed, more or less, standard growth and survival after subculture. Cells were passaged twice a week if appropriate. Otherwise, the medium was changed twice a week until the cell culture reached conditions allowing passaging. Prior to the subsequent experiments, the cells were maintained in drug-free growth medium for at least 3 weeks.

Reagents and antibodies. Irinotecan was purchased from Jiangsu Hengrui Medicine, Co., Ltd. (Jiangsu, China), 5-fluorouracil, and cisplatin were purchased from Sigma-Aldrich (St. Louis, MO, USA), dissolved in phosphate-buffered saline (PBS), curcumin (Sigma-Aldrich) was dissolved in dimethyl sulfoxide (DMSO; Sigma-Aldrich), aliquoted and stored at $4^{\circ} \mathrm{C}$ according to the manufacturer's instructions. EMT antibody sampler kit was purchased from Cell Signaling Technology (Danvers, MA, USA). Mouse monoclonal antibody against Twist1 was purchased from Santa Cruz Biotechnology (Santa Cruz, CA, USA). Rabbit polyclonal antibodies against respectively $\beta$-actin, MMP2, MMP9, CD44 and CD133 were purchased from Proteintech Group, Inc. (Rosemont, IL, USA). Rabbit polyclonal antibody against P-gp and the secondary antibodies, goat anti-rabbit IgG or goat anti-mouse IgG, were purchased from Abcam (Cambridge, MA, USA).

Cell viability assay. Cell viability assay was performed using the Cell Counting kit-8 assay (CCK-8; Dojindo Laboratories, Kumamoto, Japan). Briefly, cells (8x10\%3ell) were seeded onto a 96-well plate in $100 \mu 1 \mathrm{RPMI}-1640$ medium supplemented with $10 \% \mathrm{FBS}$ at $37^{\circ} \mathrm{C}$ in $5 \% \mathrm{CO}_{2}$. After $24 \mathrm{~h}$, drugs diluted with the culture medium were added to each well. The concentrations of the drug (irinotecan, 5-fluorouracil, cisplatin or curcumin) were $5,10,20$ and $40 \mu \mathrm{g} / \mathrm{ml}$, respectively. Following drug treatment for $24 \mathrm{~h}$, the culture medium in the 96-wells was replaced with fresh medium containing $10 \%$ CCK- 8 reagent, after $2 \mathrm{~h}$, the absorbance at $450 \mathrm{~nm}$ was measured and the values were corrected by subtracting the absorbance of blank wells that did not contain cells.

Cellular morphology and immunofluorescence assay. Cells grown on coverslips were washed three times with PBS. They were fixed for $10 \mathrm{~min}$ with cold paraformaldehyde and washed 
3 times with PBS. The coverslips were then blocked in a solution of PBS, $10 \%$ bovine serum, and $0.1 \%$ Tween-20 for $1 \mathrm{~h}$. The cells were incubated with primary antibody (E-cadherin, 1:100; vimentin, 1:100; CD44, 1:100) for $1 \mathrm{~h}$, then washed twice with PBS, and incubated with secondary antibodies diluted in PBS (Alexa Fluor 594 (red/green)-conjugated anti-rabbit IgG antibody (Santa Cruz Biotechnology) diluted 1:100 in blocking buffer). After incubating on coverslips for $1 \mathrm{~h}$ at $37^{\circ} \mathrm{C}$ the coverslips were washed as described above and mounted on slides in medium containing DAPI. Cells were observed and images were captured through an epifluorescence microscope (Olympus BX51; Olympus, Tokyo, Japan).

Lentiviruses transfection assay. Lentiviruses overexpressing human Twist1 and empty vector were built by Shanghai Genechem, Co., Ltd. (Shanghai, China). For lentiviral transfection of LoVo cells, 100 multiplicity of infection Twist1 or empty vector lentiviruses were added to a well containing $5 \times 10^{4}$ cells, medium and $8 \mu \mathrm{g} / \mathrm{ml}$ polybrene. After $24 \mathrm{~h}$ of incubation, transfected cells were selected with $2 \mu \mathrm{g} / \mathrm{ml}$ puromycin (Sigma-Aldrich). Empty vector lentivirus was used as a control. Selected cells were maintained in growth medium with $0.5 \mu \mathrm{g} / \mathrm{ml}$ puromycin.

Inhibition of Twist1 expression by RNAi. Twist1-targeted small interfering RNA (si-Twist1) (target DNA sequence is 5'-GG UACAUCGACUUCCUCUATT-3') and no-target control siRNA (NC) were purchased from Guangzhou RiboBio, Co., Ltd. (Guangzhou, China). Cells $\left(2 \times 10^{5}\right)$ were seeded in 6-well plates in triplicate, after overnight incubation, the cells were transfected with NC or si-Twist1. The siRNA (50 nmol) and Lipofectamine 3000 (Invitrogen, Waltham, MA, USA) were mixed in $100 \mu \mathrm{l}$ of minimal essential medium (Opti-MEM; Gibco) for $15 \mathrm{~min}$, and the siRNA/Lipofectamine 3000 mixture was added into the serum-free culture medium. Six hours later, the medium was replaced with complete medium, and then the cells were cultured for an additional $48 \mathrm{~h}$ prior to the detection of Twist1 expression knockdown by RT-PCR.

Migration and invasion assay. Cell migration and invasion were assessed with Boyden chambers or modified Boyden chambers according to the protocol of the manufacturer (Becton-Dickinson Labware, Bedford, MA, USA). Briefly, $100 \mu 1$ RPMI-1640 medium without FBS included 100,000 cells was placed on an $8.0-\mu \mathrm{m}$ pore size membrane insert in 24-well plates, and $600 \mu 1$ RPMI-1640 medium with 10\% FBS was placed in the bottom wells. After $24 \mathrm{~h}$ at $37^{\circ} \mathrm{C}$, cells that did not migrate were removed from the top side of the inserts with a cotton swab. After fixed with $4 \%$ paraformaldehyde for $10 \mathrm{~min}$, cells that had migrated to the underside of the inserts were stained with $0.1 \%$ crystal violet solution and the cells on each insert were counted using a microscope (Olympus BX51; Olympus). Cells were counted in five random fields per insert. The invasion assay was done in a similar fashion except the 8.0- $\mu \mathrm{m}$ pore size membrane inserts were coated with Matrigel. Results were expressed as cells migrated per field.

Quantitative real-time PCR assay. Total mRNA of the cells was extracted using the Total RNA kit (TransGen Biotech, Inc., Beijing, China). First step cDNA synthesis was generated from
$500 \mathrm{ng}$ total mRNA. RT-PCR amplification was performed in triplicate on LightCycler ${ }^{\circledR} 480$ II (Roche, Berlin, Germany). The primers used in each reaction were synthesized in Sangon Biotech, Co., Ltd. (Shanghai, China). Expression levels for each target gene were normalized to $\beta$-actin gene. Results were calculated using the comparative threshold cycle $(\Delta \Delta \mathrm{CT})$ method. Data are presented as the mean \pm standard deviation (SD) from three independent experiments.

Western blotting assay. Cells were harvested with a plastic scraper and washed twice with cold PBS. Cells were then lysed with ice-cold protein extract solution RIPA (Beyotime Institute of Biotechnology, Shanghai, China) and protein concentration was quantified using the BCA procedure (Beyotime Institute of Biotechnology). Equal amounts $(30 \mu \mathrm{g})$ of protein samples were separated by SDS-PAGE using $12 \%$ or $10 \%$ polyacrylamide gel and then transferred onto a PVDF membrane (Millipore, Billerica, MA, USA). The membrane was blocked with 5\% skim milk TBST (Tris-buffered saline Tween-20) buffer for $1 \mathrm{~h}$ at room temperature. The membranes were incubated with primary antibodies including EMT antibody sampler kit (1:1,000), anti-Twist1, anti-MMP1, antiMMP9, anti-CD44, anti-CD133 (1:500); anti-P-gp, anti- $\beta$-actin $(1: 3,000)$ at $4^{\circ} \mathrm{C}$ overnight. After washed with TBST, the blot was incubated with the appropriate horseradish peroxidaseconjugated anti-rabbit or anti-mouse secondary antibodies $(1: 5,000)$. The antigen was detected by WesternBright ECL HRP substrate kit (Millipore) in gel image analysis system (Kodak, Rochester, NY, USA). The intensities of the protein bands were analyzed by Molecular Imaging software. $\beta$-actin protein was used as the internal control.

Statistical analysis. Quantitative data are expressed as the mean \pm SD of three independent experiments. Comparisons among multiple groups were performed using one-way analysis of variance (ANOVA) test followed by Dunnett's test. P-values are two-sided, and a value of 0.05 was considered to be statistically significant. All statistical calculations were performed using SPSS software (version 19.0; SPSS, Inc., Chicago, IL, USA). Graphs were prepared using GraphPad Prism 5.

\section{Results}

Cross-resistance to anticancer drugs and increased expression of $A B C B 1$ in irinotecan-resistant cells. Via long-term culturing of original irinotecan-sensitive LoVo cells by gradual adaptation of increasing irinotecan concentrations, we successfully established irinotecan-resistant variant $\mathrm{LoVo} /$ CPT-11R cells. The resistant cells were capable of longterm survival and proliferation in media containing $70 \mu \mathrm{g} /$ $\mathrm{ml}$ irinotecan. In order to investigate whether the LoVo/ CPT-11R cells acquired cross-resistance to other anticancer drugs, their sensitivities to various anticancer drugs used to treat CRC, including 5-fluorouracil, cisplatin and curcumin, were determined using CCK-8 assay. The results showed that cell viabilities of LoVo/CPT-11R cells treated with anticancer drugs (irinotecan, 5-fluorouracil, cisplatin and curcumin) were markedly increased compared with the control LoVo cells (Fig. 1A-D), herein the LoVo/CPT-11R cells acquired multidrug resistance property. 

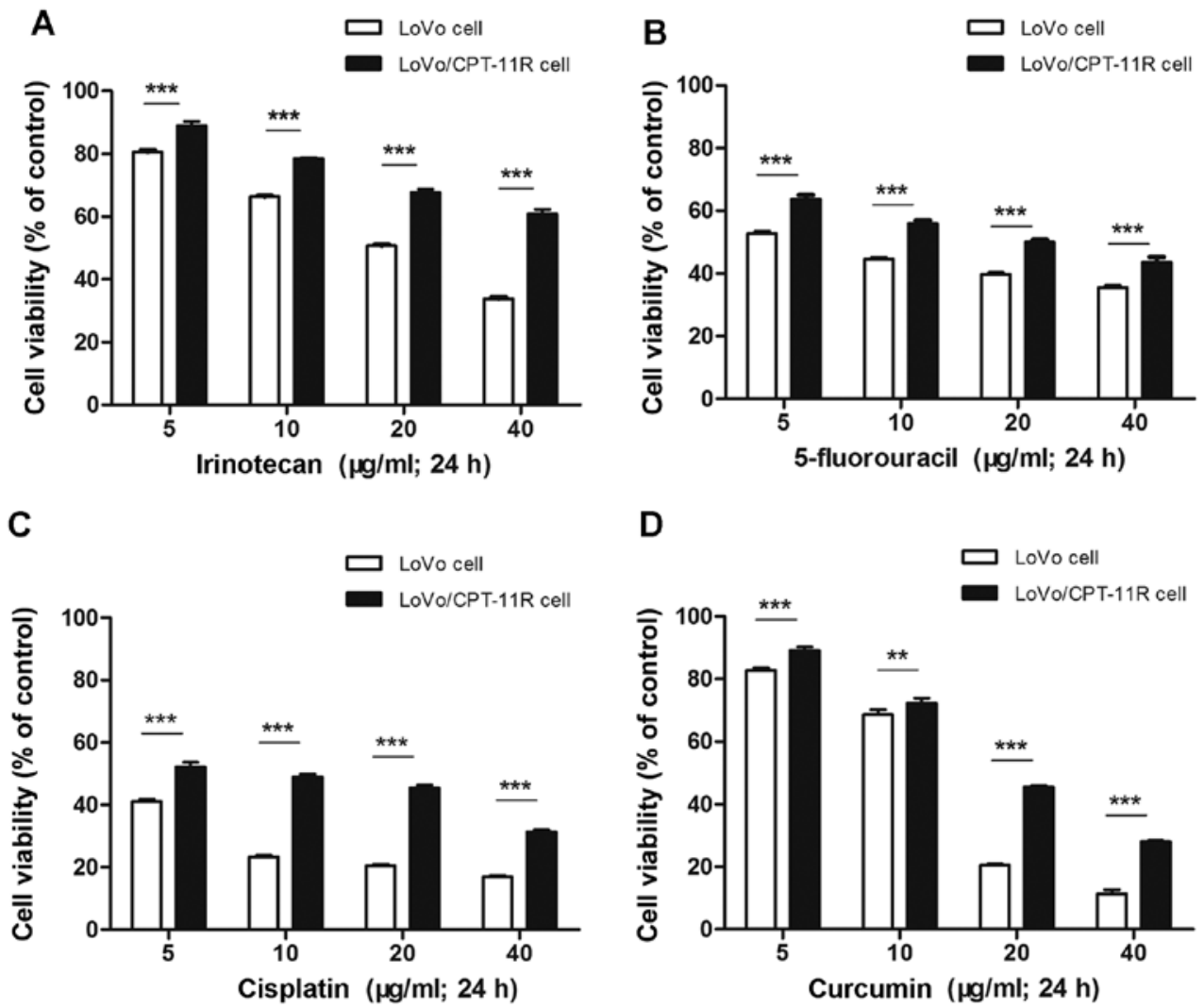

$\mathbf{E}$

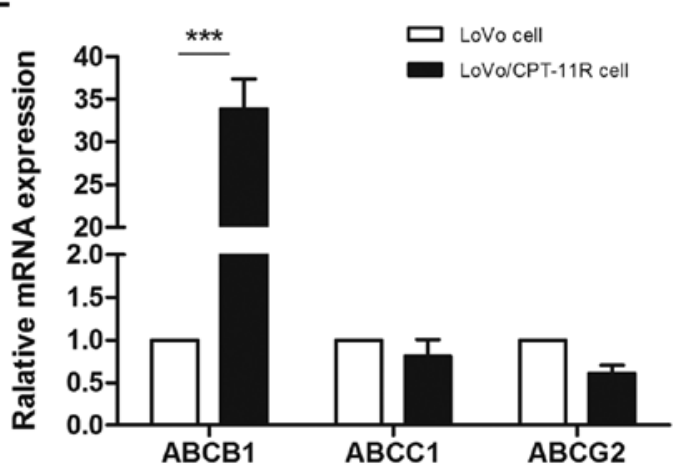

$\mathbf{F}$

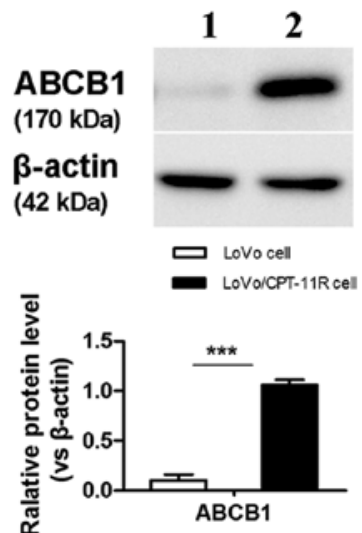

Figure 1. Cell viability to anticancer drugs and expression levels of $A B C$ transports in LoVo/CPT-11R cells. (A-D) LoVo and LoVo/CTP-11R cells were treated with various concentrations of (A) irinotecan, (B) 5-fluorouracil, (C) cisplatin, or (D) curcumin for $24 \mathrm{~h}$, and cell viabilities were determined using CCK-8 assay. (E) The mRNA expression of ABCB1, ABCC1 and ABCG2 in LoVo cells and LoVo/CPT-11R cells were analyzed by qRT-PCR assay. (F) ABCB1 protein (P-gp) expression was detected by western blotting assay. $(\mathrm{G})$ Relative ABCB1 protein expression was measured by ImageJ. $\beta$-actin was used as a loading control. Each bar represents the mean $\pm \mathrm{SD}$ of the three independent experiments. ${ }^{* *} \mathrm{P}<0.01,{ }^{* * *} \mathrm{P}<0.001$ vs. LoVo cells. Lane 1 , LoVo cells; lane 2 , LoVo/CPT-11R cells.

Additionally, we compared expression of the most known $\mathrm{ABC}$ transporter genes (ABCB1, ABCC1 and ABCG2) in mRNA level in LoVo and LoVo/CPT-11R cells. Results indicated that mRNA expression of ABCB1 was significantly increased in LoVo/CPT-11R cells, but the changes of expression of $\mathrm{ABCC} 1$ and $\mathrm{ABCG} 2$ were not significant (Fig. 1E). In addition, the expression of $\mathrm{ABCB} 1$ protein (P-gp) was detected by western blotting assay. Likewise, the $\mathrm{ABCB} 1$ was significantly overexpressed on protein level in LoVo/CPT-11R cells (Fig. 1F and G).

EMT-like morphology and altered localization of EMT markers in irinotecan-resistant cells. Light microscopy revealed a marked alteration of cellular morphology in monolayer culture. LoVo/CPT-11R cells exhibited spindle shape, loose intercellular space, and irregular scattering, suggested a more 'mesenchymal' phenotype than parental LoVo cells (Fig. 2A). To further confirm our observation, the EMT marker proteins were stained by immunofluorescence assay. It was found that epithelial marker E-cadherin was located mainly in cell membrane of LoVo cells, but E-cadherin protein was located mainly in the cytoplasm of LoVo/CPT-11R cells (Fig. 2B). Moreover, compared to LoVo cells, the mesenchymal marker Vimentin protein was more distinctly stained in cytoskeletal localization in LoVo/CPT-11R cells (Fig. 2C). 
A

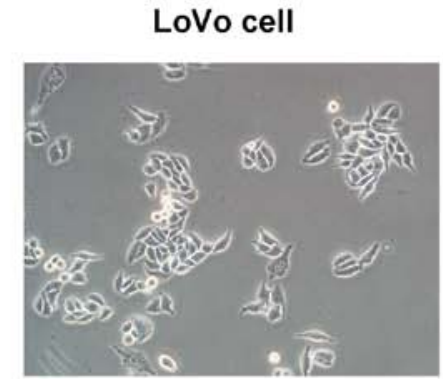

B
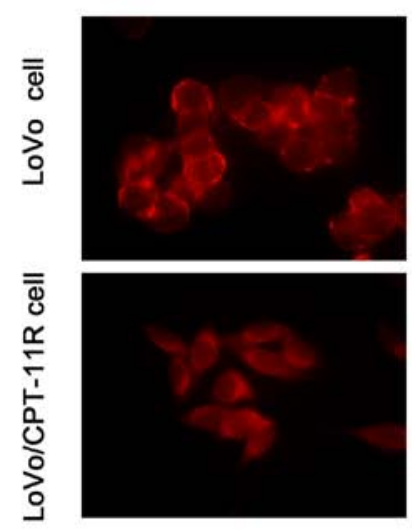

C
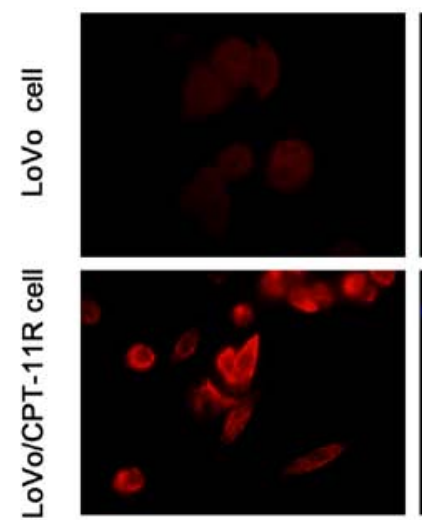

DAPI
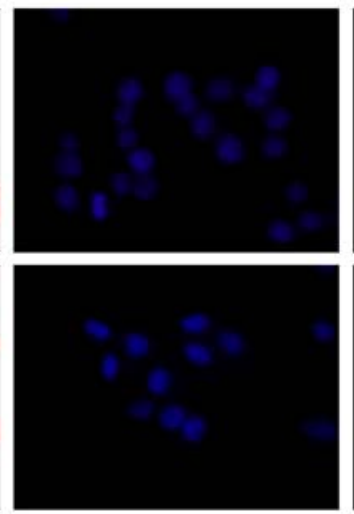

DAPI
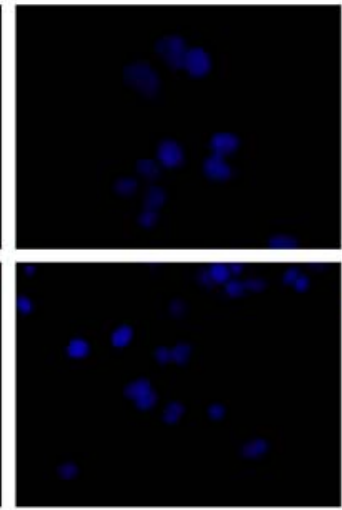

LoVo/CPT-11R cell

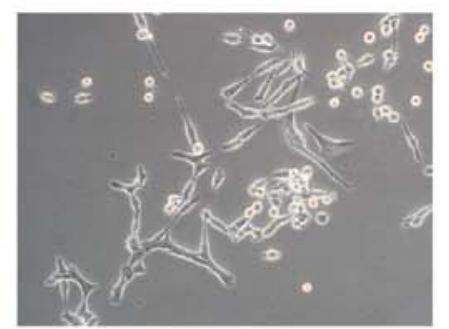

Merge
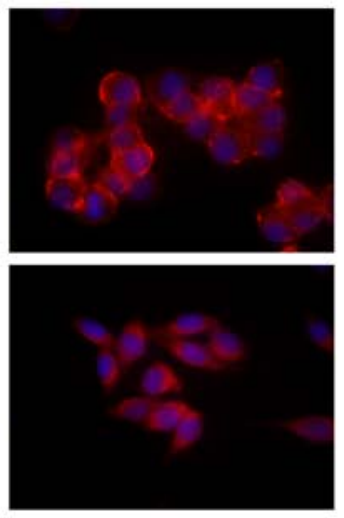

Merge
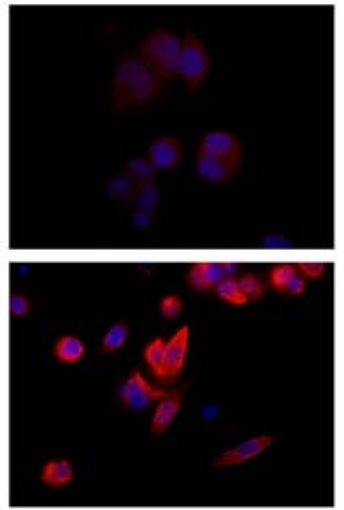

Figure 2. Cellular morphology and altered localization of EMT markers. (A) Cellular morphology was detected by Light microscopy in monolayer culture. (Original magnification, x200). Cellular localization of (B) E-cadherin and (C) vimentin were determined by immunocytochemistry assay in LoVo cells and LoVo/CPT-11R cells. Cells were stained with DAPI (blue) and second antibody (red). Fluorescent images were captured under an epifluorescence microscope. (Original magnification, x1000).

Irinotecan-resistant cells overexpress EMT markers, Twist1 and CSC markers. The observed morphological changes implied that the irinotecan-resistant cells had transitioned to a mesenchymal phenotype. To determine whether these morphological changes were associated with EMT, we examined respectively the expression of EMT markers in mRNA and protein levels. Consistent with morphological changes, LoVo/CPT-11R cells showed downregulation of the epithelial markers E-cadherin and ZO1, and upregulation of the mesenchymal markers vimentin and $\mathrm{N}$-cadherin (Fig. 3A-C). These results suggested that EMT could be associated with acquired drug resistance after long-term exposure to irinotecan. In addition, EMT-inducing transcription factors were also detected by the above methods. The expression of Twist 1 was higher in LoVo/CPT-11R cells than LoVo cells, the changes of expres- sion of Snail, Slug and Zeb1 were not statistically significant (Fig. 3D-F).

The relation of aggressive cell characteristics and CSC continue to be under intense investigation. Therefore, we detected respectively the expression of CSC identification markers. It was founded that the expression of CD44 and CD133, most widely studied CSC biomarkers, were increased in mRNA and protein levels in LoVo/CPT-11R cells compared to LoVo cells (Fig. 3G-I).

Overexpression of Twist 1 contributes to irinotecan resistance, EMT and CSC-like phenotype of LoVo cells. Considering the expression of Twist 1 was significantly increased in LoVo/CPT-11R cells, we built overexpressed Twist1 LoVo cells by lentivirus transfection assay, to confirm whether 
A

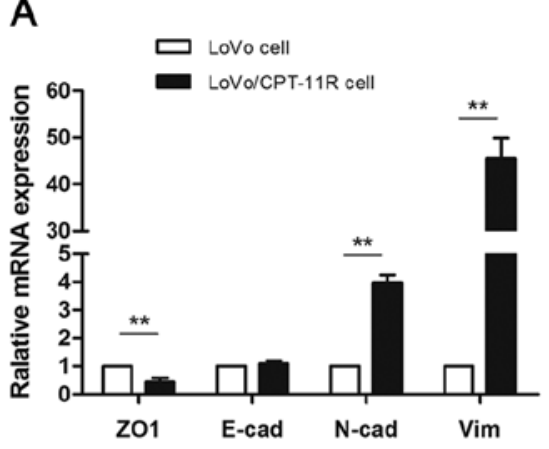

D

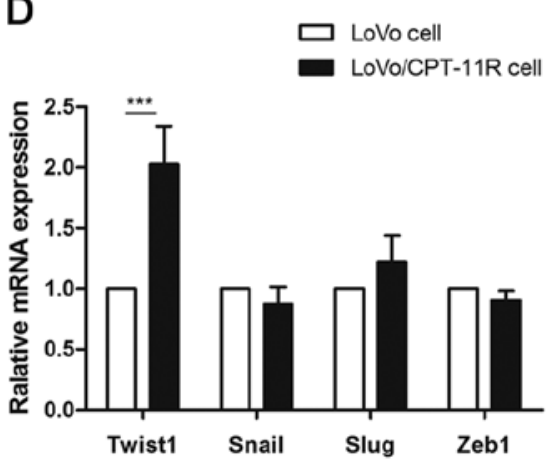

G $\square$ LoVo cell
LoVo/CPT-11R cell

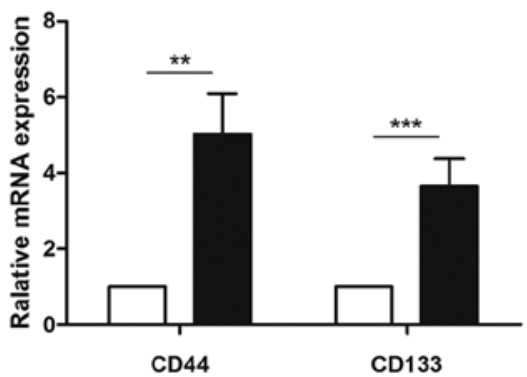

B

ZO1

(194 kDa)

E-cadherin

(135 kDa)

$\mathrm{N}$-cadherin

(140 kDa)

Vimentin

(57 kDa)

$\beta$-actin

$(42 \mathrm{kDa})$

$E$

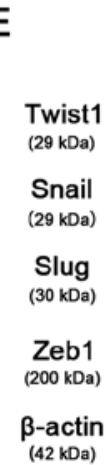

$\mathrm{H}$
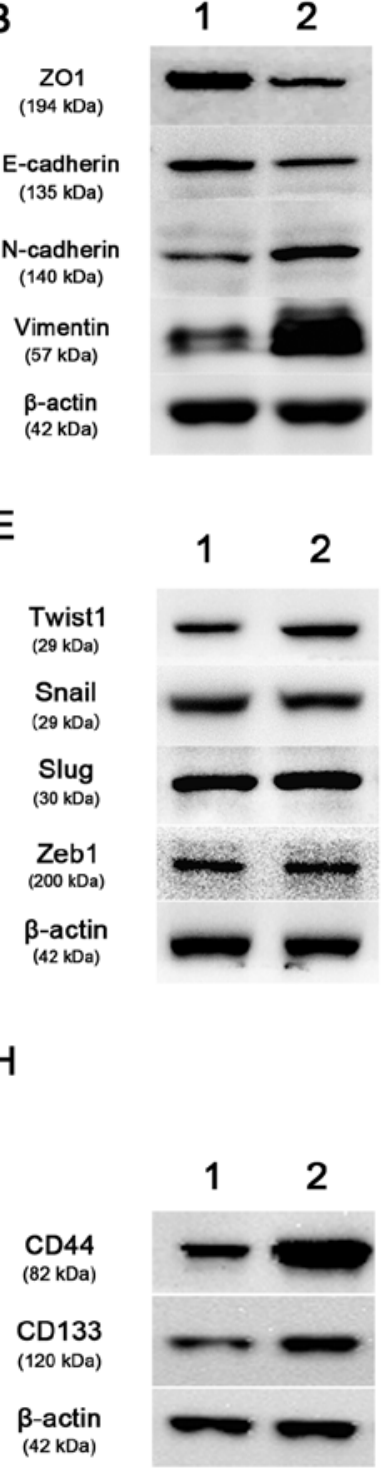

C

F
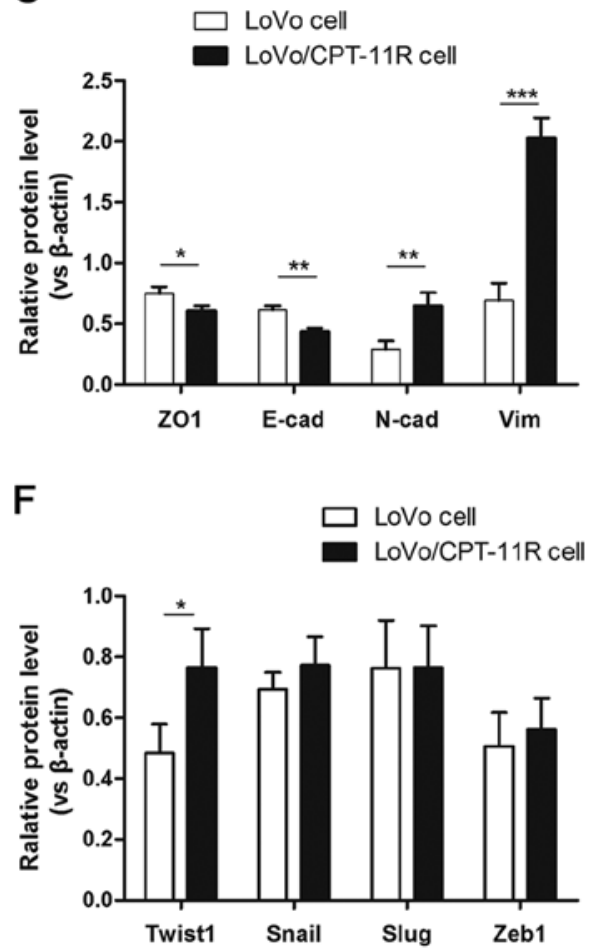

I

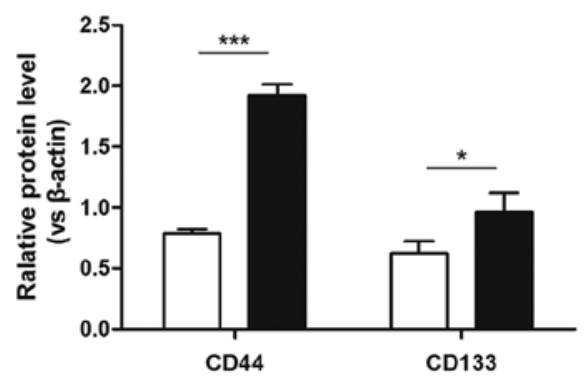

Figure 3. Expression of EMT markers, EMT-inducing transcription factors and CSC markers. The mRNA expression levels of EMT markers (A), EMTinducing transcription factors (D) and CSC markers (G) were analyzed by qRT-PCR assay in LoVo cells and LoVo/CPT-11R cells. Protein expression and relative protein expression levels of EMT markers (B and C), EMT-inducing transcription factors (E and F) and CSC markers (H and I) were detected by western blotting assay and measured by Image J. $\beta$-actin was used as a loading control. Values present the mean \pm SD of three independent experiments. ${ }^{*} \mathrm{P}<0.05,{ }^{* *} \mathrm{P}<0.01,{ }^{* * *} \mathrm{P}<0.001$ vs. LoVo cells. Lane 1, LoVo cells; lane 2, LoVo/CPT-11R cells. E-cad, E-cadherin; N-cad, N-cadherin; Vim, vimentin.

overexpression of Twist1 contributed to resistance to irinotecan, EMT and CSC-like phenotype of LoVo cells. The control LoVo cells (transfected with empty vector lentiviruses) and LoVo/Twist1 cells (transfected with overexpressed Twist1 lentiviruses) were treated with different concentrations of irinotecan and CCK- 8 assays were performed. The results showed that overexpression of Twist1 led to a distinct increase in cell viability compared with control cells (Fig. 4A). Moreover, we detected respectively the expression of EMT and CSC markers in protein level by western blotting assay. The results showed that the expression of E-cadherin was decreased and that the expression of CD44 was increased (Fig. 4B and C).

Overexpression of Twistl enhances migration and invasion potential by regulating MMP2 expression. To assess the migratory and invasive potentials of overexpressed Twist1 LoVo cells, in vitro cell migration and invasion assay were performed. Compared with the controls, LoVo/Twist1 cells demonstrated a 1.74 -fold increase at $24 \mathrm{~h}$ in migration (Fig. 5A and B), and a 2.63-fold increase at $48 \mathrm{~h}$ in invasion (Fig. 5C and D). Metalloproteinases (MMPs) are crucial to invasion and migration, a significant association has been reported between tumor aggressiveness and increased levels of MMP2 and MMP9 in many experimental and clinical studies. Our results detected that the expression of MMP2 was significantly increased in the protein level, but the MMP9 was not significantly changed in LoVo/Twist1 cells compared to control cells.

Downregulation of Twist1 increases the irinotecan sensitivity, and reverses EMT and CSC-like phenotype of LoVo/CPT-11R 
A
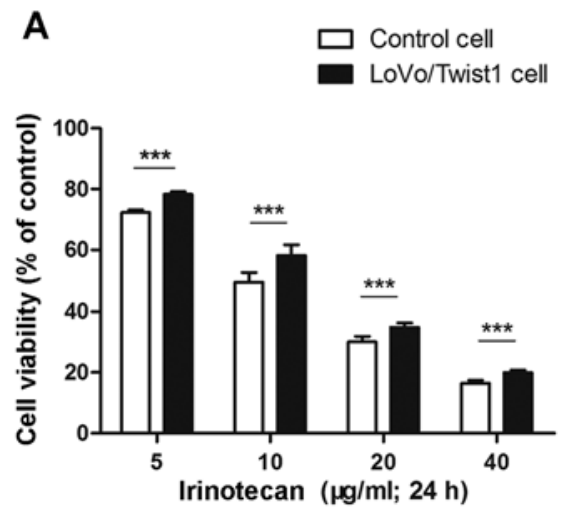

B

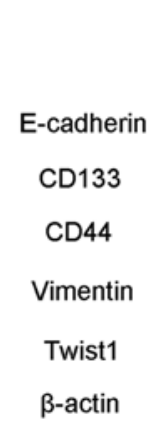

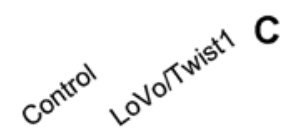

C<smiles>[CH]C</smiles>

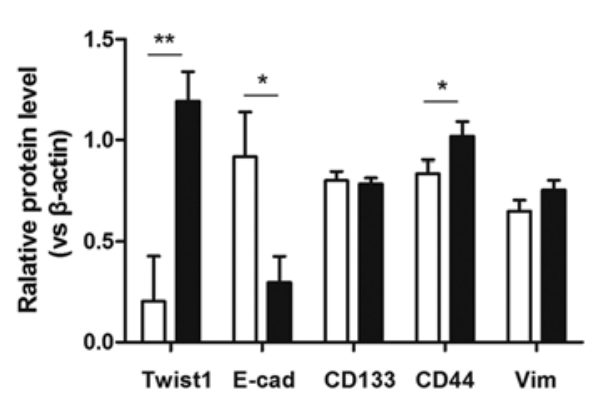

Figure 4. Overexpression of Twist decreases the sensitivity to irinotecan and induces EMT and CSC-like phenotype of LoVo cells. (A) The control cells and $\mathrm{LoVo} /$ Twistl cells were respectively treated with various concentrations of irinotecan $(5,10,20$ and $40 \mu \mathrm{g} / \mathrm{ml}) \mathrm{for} 24 \mathrm{~h}$, and cell viabilities were determined by CCK-8 assay. (B and C) Protein expression of EMT and CSC markers were detected by western blotting assay and relative protein expression levels measured by ImageJ. $\beta$-actin was used as a loading control. Each bar represents the mean $\pm \mathrm{SD}$ of the three independent experiments. ${ }^{*} \mathrm{P}<0.05,{ }^{* * *} \mathrm{P}<0.01,{ }^{* * *} \mathrm{P}<0.001$ vs. LoVo cells. Control, LoVo cells transfected with empty vector lentiviruses; LoVo/Twist1, overexpressed Twist1 LoVo cells.

A

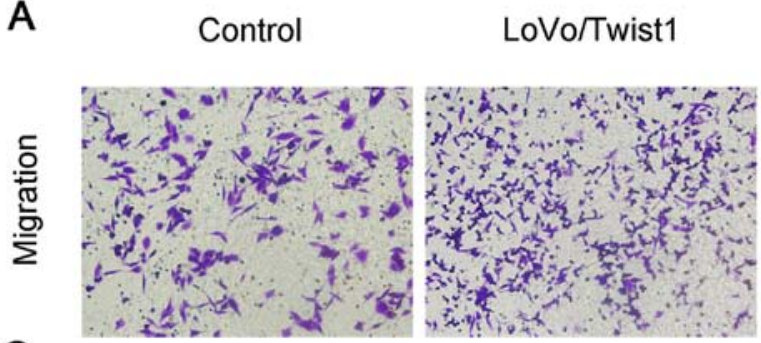

C

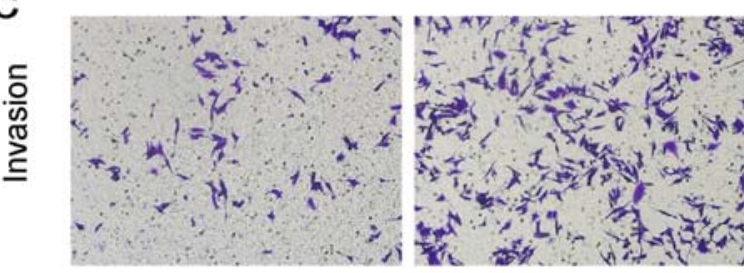

B

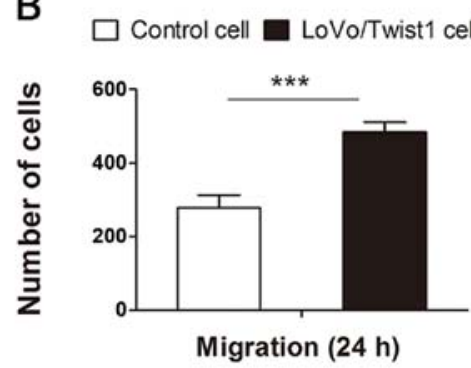

D

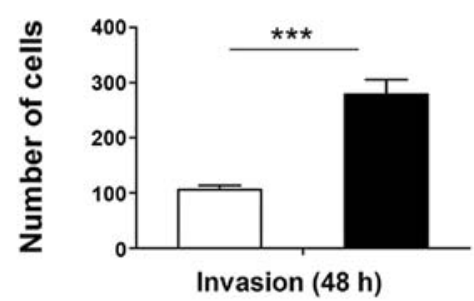

$\mathrm{E}$

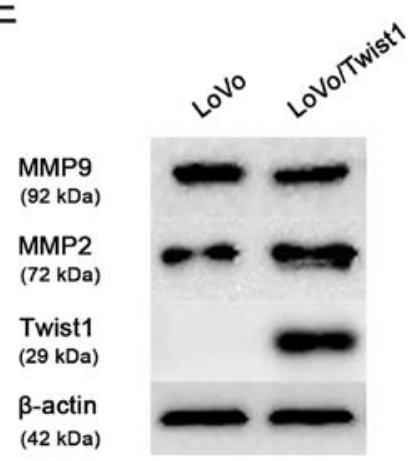

$\mathrm{F}$

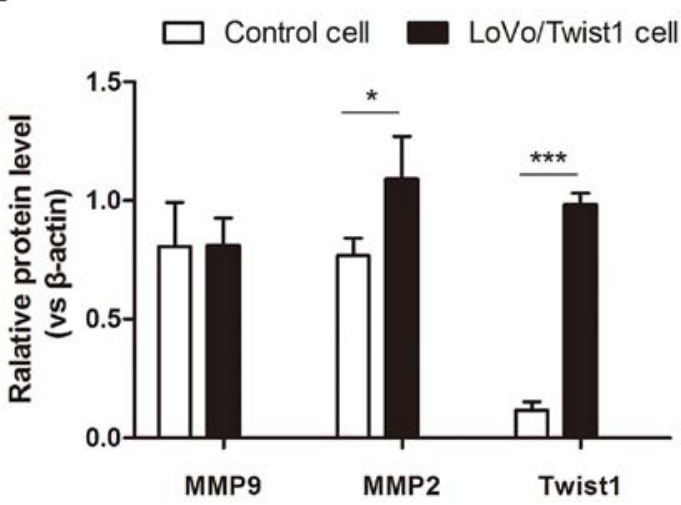

Figure 5. Overexpression of Twist1 enhances migration and invasion potential and increases MMPs expression. (A and B) The number of overexpressed Twist1 LoVo cells are 1.74-fold increased at $24 \mathrm{~h}$ in migration. (C and D) The number of overexpressed Twist1 LoVo cells are 2.63 -fold increased at $48 \mathrm{~h}$ in invasion. (Original magnification, x200). (E and F) The expression of Twist1, MMP2 and MMP9 protein in overexpressed Twist1 LoVo cells compared to control cells. Values are presented as the mean $\pm \mathrm{SD}$ of three independent experiments. ${ }^{*} \mathrm{P}<0.05,{ }^{* * *} \mathrm{P}<0.001$ vs. control cells. Control, LoVo cells transfected with empty vector lentiviruses; LoVo/Twist1, overexpressed Twist1 LoVo cells.

cells. To furtherly discover the molecular link between irinotecan resistance and Twist1, we inhibited the expression of
Twist1 in LoVo cells and LoVo/CPT-11R cells by RNAi. After transfected respectively with NC and si-Twistl in LoVo cells 

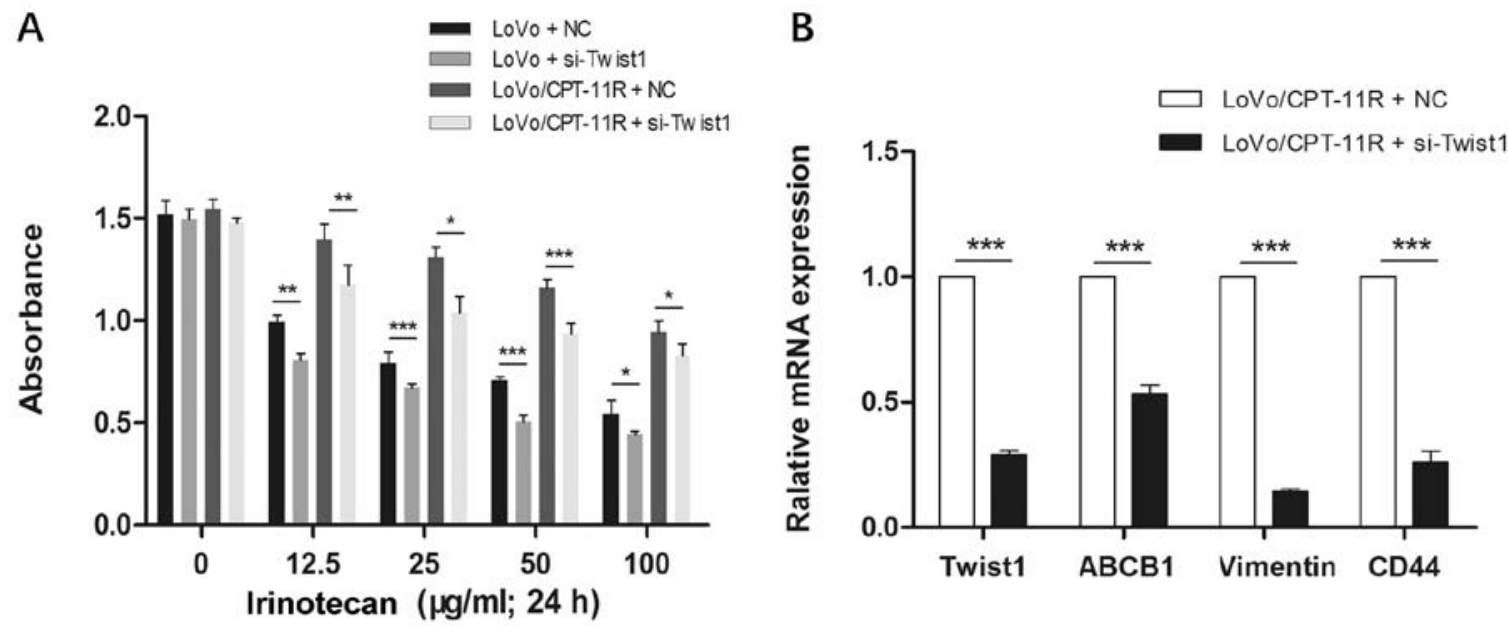

C

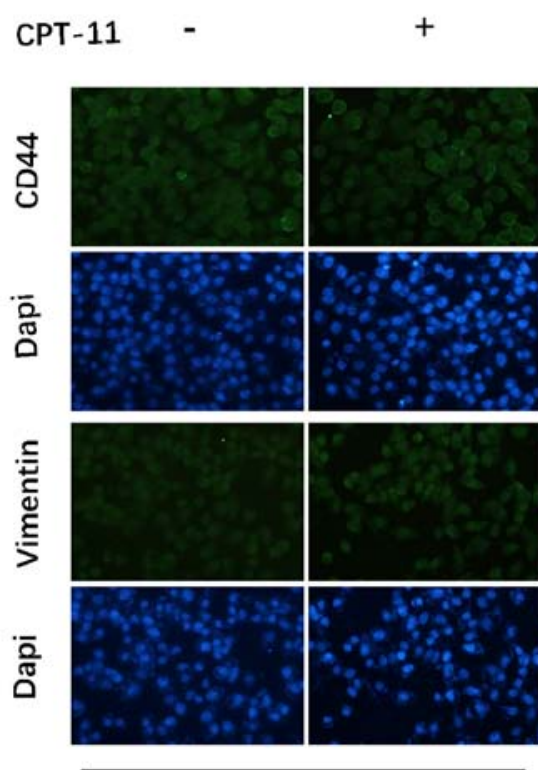

LoVo + NC

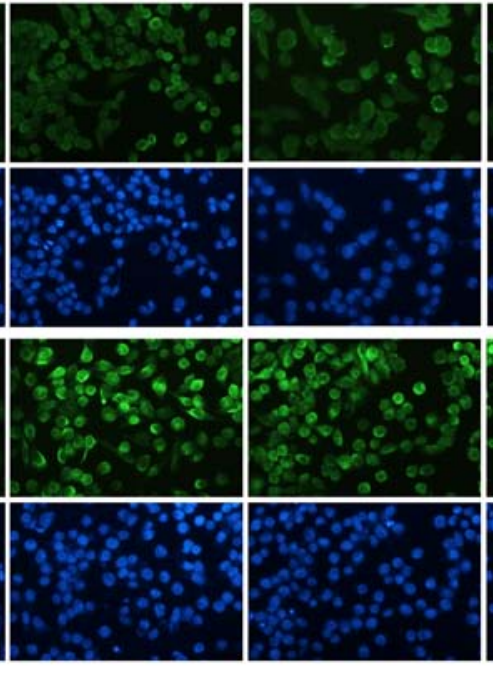

LoVo/CPT-11R + NC

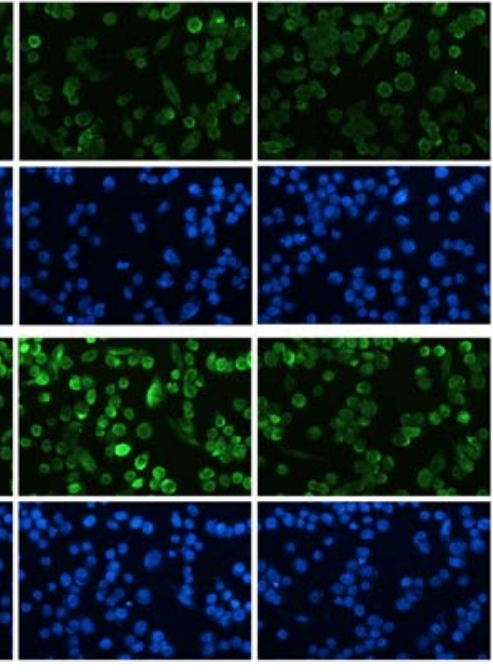

LoVo/CPT-11R + si-Twist1

Figure 6. Downregulation of Twist1 increases the sensitivity to irinotecan and reverses EMT and CSC-like phenotype of the LoVo/CPT-11R cells. (A) After transfected respectively with NC and Twist1-targeted siRNA in LoVo cells and LoVo/CPT-11R cells, cells were treated with different concentrations of irinotecan $(0,12.5,25,50$ and $100 \mu \mathrm{g} / \mathrm{ml})$ for $24 \mathrm{~h}$, then every group absorbance at $450 \mathrm{~nm}$ was detected by CCK- 8 assays. (B) The mRNA expression of Twist1, ABCB1, vimentin and CD44 were analyzed by qRT-PCR assay. (C) The protein expression of vimentin and CD44 were detected by immunocytochemistry assay. Cells were stained with Dapi (blue) and second antibody (green). (Original magnification, x200). Each bar represents the mean \pm SD of the three independent experiments. ${ }^{*} \mathrm{P}<0.05,{ }^{* *} \mathrm{P}<0.01,{ }^{* * * *} \mathrm{P}<0.001$. NC, cells transfected with $\mathrm{N}$ Control; si-Twist1, cells transfected with Twist1-targeted siRNA.

and $\mathrm{LoVo} / \mathrm{CPT}-11 \mathrm{R}$ cells, cells were treated with different concentrations of irinotecan $(0,12.5,25,50$ and $100 \mu \mathrm{g} / \mathrm{ml})$ for $24 \mathrm{~h}$, then absorbance of each group at $450 \mathrm{~nm}$ was detected by CCK-8 assays. The results showed that the inhibition of Twist1 expression led to a distinct decrease in absorbance (Fig. 6A). It indicated that the downregulation of Twistl could increase the irinotecan sensitivity in LoVo cells and LoVo/ CPT-11R cells.

Moreover, we detected respectively the expression of EMT and CSC-like markers by RT-PCR and immunofluorescence assays. The results showed that the inhibition of Twist1 could downregulate the mRNA expression of vimentin, CD44 and P-gp (Fig. 6B), and distinctly decreased the protein expression of vimentin and CD44 of LoVo/CPT-11R cells when adding irinotecan (Fig. 6C). It indicated that the downregulation of Twist1 could reverse EMT and CSC-like phenotype of LoVo/CPT-11R cells.

\section{Discussion}

We established irinotecan-resistant subline of human colon cancer LoVo cells by stepwise adaptation to increasing drug concentrations. In addition, $\mathrm{LoVo} / \mathrm{CTP}-11 \mathrm{R}$ cells also displayed cross-resistance to non-camptothecin anticancer drugs (5-fluorouracil, cisplatin and curcumin), indicated the presence of the multidrug resistant (MDR) phenotype. Numerous published studies have showed that ATP-binding cassette (ABC) transporters play a crucial role in the development of multidrug resistance by the efflux of anticancer agents outside the cancer cells (24). The most extensively characterized 
MDR transporters included ABCB1 (also known as MDR1 or P-glycoprotein), ABCC1 (also known as MRP1) and ABCG2 (also known as BCRP or MXR) (25). When compared with the genes expression in LoVo cells, we found only the ABCB1 was significantly higher in LoVo/CPT-11R cells, and the expression of P-gp was also increased. Indeed, ABCB1 encoded the xenobiotic transporter P-gp that has been extensively investigated in vitro and in vivo as a predictor of MDR in various tumors $(26,27)$. The expression of ABCB1 is regulated at different levels by multiple signaling pathways, including hypoxia-inducible factor- $1 \alpha$ (HIF-1 $\alpha)$, p53, chromosomal rearrangement, methylation, acetylation and microRNA (28). Recently, a potential transcriptional regulatory role of Twist1 has been identified in chemotherapy drug resistance (29). In the present study, the positively correlated downregulation of ABCB1 and Twist1 in LoVo/CPT-11R cells was detected, which also indicate a novel role of Twist1 in maintaining the irinotecan-resistant phenotype of colon cancer through regulating $\mathrm{ABCB} 1$ expression.

EMT program is now known to facilitate the metastatic spread and progression of cancer cells from the site of the primary tumor to the surrounding tissues and distant organ(s). It is also generally considered that EMT is associated with cancer aggressiveness, invasive and metastatic behavior, and chemotherapeutic resistance (30). However, comprehensive studies of EMT and irinotecan resistance in colon cancer are lacking. In the present study, we found that LoVo/CPT-11R cells exhibited a more mesenchymal phenotype and location alteration of epithelial marker E-cadherin from cell membrane to cytoplasm than parental LoVo cells. To confirm the role of EMT and resistance to irinotecan, we examined the expression of EMT markers in mRNA and protein levels, respectively. As expected, LoVo/CPT-11R cells showed downregulation of E-cadherin and $\mathrm{ZO} 1$ and upregulation of vimentin and $\mathrm{N}$-cadherin. In addition, the expression levels of EMT-inducing transcription factors (Twist1, Snail, Slug and Zeb1) were detected. Notably, results showed that only Twist1 was significantly higher in LoVo/CPT-11R cells than LoVo cells. Increasing evidence suggested that EMT not only enables cancer cells to disseminate but also to acquire the ability to self-renew by inducing a CSC trait (31-33). In experimental models, CSCs are more resistant than differentiated tumor cells to chemo- and radiotherapy and they can escape from the effects of conventional cytotoxic treatments (34). It has been reported that CD133 and CD44 are two proposed stem cell markers in various metastasized cancers including colorectal cancer (35-37). Therefore, we detected respectively the expression of CSC identification markers. The expression of CD44 and CD133 were increased both in mRNA and protein levels in LoVo/CPT-11R cells. Therefore, it was identified that the irinotecan resistance cells presented EMT-like and CSC-like phenotype. In order to confirm whether Twistl contributes to formation of resistance to irinotecan and alteration of EMT, CSC-like phenotype in colon cancer cells, we established overexpressed Twist1 LoVo cells, named LoVo/Twist1 cells, by lentivirus transfection assay. After treated with different concentrations of irinotecan, we found that the overexpression of Twist1 led to a distinct resistance to irinotecan compared with control cells transfected with empty vector. Moreover, the decreased expression of E-cadherin and the increased expression of CD44 demonstrated the formation of EMT, CSC-like phenotype in LoVo/Twist1 cells. In contrast, we found that downregulation of Twist1 increased the irinotecan sensitivity, reversed EMT and CSC-like phenotype of LoVo/ CPT-11R cells transfected with NC and si-Twist1. Therefore, it was identified that EMT and CSC-like phenotype induced by Twist 1 contribute to acquire resistance to irinotecan.

In addition, compared with the controls, LoVo/Twist1 cells performed a significant enhancement both in migration and invasion. It is known that MMPs are a group of matrix-degrading proteins implicated in several pathological processes, including invasion and metastasis of CRC (38). Increased MMP2 and MMP9 expression have also been documented to correlate with cancer invasion (39). Consistent with previous reports, MMP2 protein was increased in LoVo/Twist1 cells, but MMP9 was not significantly changed compared to control cells, which indicated that overexpressed Twist 1 contribute to the migratory and invasive potentials of colon cancer by upregulated MMP2 expression. In summary, the development of a highly aggressive colon cancer phenotype requires the coordination of many different molecular changes, which are a consequence of genomic alterations. We have demonstrated that EMT and CSC-like phenotype induced by Twist1 contribute to acquiring resistance to irinotecan, and high expression of Twist1 enhanced migration and invasion potential. It suggests that Twist1 may be a potential molecular target for overcoming the irinotecan resistance and metastasis in colon cancer. However, it would make the resistance mechanisms associated with Twist1 more fruitful if gene expression profiling of LoVo/CPT-11R cells was added to the analysis.

\section{Acknowledgements}

The present study was supported by the China Postdoctoral Science Foundation Grant to G.W. (grant no. 2015M582358) and the Project of Traditional Chinese Medicine Bureau of Guang Dong Province to X.C. (grant no. 20141307).

\section{References}

1. Arnold M, Sierra MS, Laversanne M, Soerjomataram I, Jemal A and Bray F: Global patterns and trends in colorectal cancer incidence and mortality. Gut 66: 683-691, 2017.

2. Cao H, Xu E, Liu H, Wan L and Lai M: Epithelial-mesenchymal transition in colorectal cancer metastasis: A system review. Pathol Res Pract 211: 557-569, 2015.

3. Panczyk M: Pharmacogenetics research on chemotherapy resistance in colorectal cancer over the last 20 years. World J Gastroenterol 20: 9775-9827, 2014.

4. Sanz-Garcia E, Grasselli J, Argiles G, Elez ME and Tabernero J: Current and advancing treatments for metastatic colorectal cancer. Expert Opin Biol Ther 16: 93-110, 2016.

5. Sievers CK, Kratz JD, Zurbriggen LD, LoConte NK, Lubner SJ, Uboha N, Mulkerin D, Matkowskyj KA and Deming DA: The multidisciplinary management of colorectal cancer: Present and future paradigms. Clin Colon Rectal Surg 29: 232-238, 2016.

6. Van Cutsem E, Cervantes A, Adam R, Sobrero A, Van Krieken JH, Aderka D, Aranda Aguilar E, Bardelli A, Benson A, Bodoky G, et al: ESMO consensus guidelines for the management of patients with metastatic colorectal cancer. Ann Oncol 27: 1386-1422, 2016.

7. Ramesh M, Ahlawat P and Srinivas NR: Irinotecan and its active metabolite, SN-38: Review of bioanalytical methods and recent update from clinical pharmacology perspectives. Biomed Chromatogr 24: 104-123, 2010. 
8. Xu Y and Villalona-Calero MA: Irinotecan: Mechanisms of tumor resistance and novel strategies for modulating its activity. Ann Oncol 13: 1841-1851, 2002.

9. $\mathrm{Hu} \mathrm{T}, \mathrm{Li} \mathrm{Z}$, Gao $\mathrm{CY}$ and Cho $\mathrm{CH}$ : Mechanisms of drug resistance in colon cancer and its therapeutic strategies. World J Gastroenterol 22: 6876-6889, 2016.

10. Zubeldia IG, Bleau AM, Redrado M, Serrano D, Agliano A, Gil-Puig C, Vidal-Vanaclocha F, Lecanda J and Calvo A Epithelial to mesenchymal transition and cancer stem cell phenotypes leading to liver metastasis are abrogated by the novel TGF 31 -targeting peptides P17 and P144. Exp Cell Res 319: $12-22,2013$.

11. Singh A and Settleman J: EMT, cancer stem cells and drug resistance: An emerging axis of evil in the war on cancer. Oncogene 29: 4741-4751, 2010

12. Mitra A, Mishra L and Li S: EMT, CTCs and CSCs in tumor relapse and drug-resistance. Oncotarget 6: 10697-10711, 2015.

13. Bao S, Wu Q, McLendon RE, Hao Y, Shi Q, Hjelmeland AB, Dewhirst MW, Bigner DD and Rich JN: Glioma stem cells promote radioresistance by preferential activation of the DNA damage response. Nature 444: 756-760, 2006.

14. Khan MA, Chen HC, Zhang D and Fu J: Twist: A molecular target in cancer therapeutics. Tumour Biol 34: 2497-2506, 2013.

15. Zhu DJ, Chen XW, Zhang WJ, Wang JZ, Ouyang MZ, Zhong Q and Liu CC: Twist1 is a potential prognostic marker for colorectal cancer and associated with chemoresistance. Am J Cancer Res 5 : 2000-2011, 2015

16. Cheng GZ, Chan J, Wang Q, Zhang W, Sun CD and Wang LH: Twist transcriptionally up-regulates AKT2 in breast cancer cells leading to increased migration, invasion, and resistance to paclitaxel. Cancer Res 67: 1979-1987, 2007.

17. Niu RF, Zhang L, Xi GM, Wei XY, Yang Y, Shi YR and Hao XS Up-regulation of Twist induces angiogenesis and correlates with metastasis in hepatocellular carcinoma. J Exp Clin Cancer Res 26: 385-394, 2007.

18. Ji H, Lu HW, Li YM, Lu L, Wang JL, Zhang YF and Shang H: Twist promotes invasion and cisplatin resistance in pancreatic cancer cells through growth differentiation factor 15 . Mol Med Rep 12: 3841-3848, 2015.

19. Lee KW, Kim JH, Han S, Sung CO, Do IG, Ko YH, Um SH and Kim SH: Twist1 is an independent prognostic factor of esophageal squamous cell carcinoma and associated with its epithelial-mesenchymal transition. Ann Surg Oncol 19: 326-335, 2012.

20. Chanmee T, Ontong P, Mochizuki N, Kongtawelert P, Konno K and Itano N: Excessive hyaluronan production promotes acquisition of cancer stem cell signatures through the coordinated regulation of Twist and the transforming growth factor $\beta$ (TGF- $\beta$ )-Snail signaling axis. J Biol Chem 289: 26038-26056, 2014.

21. Li J and Zhou BP: Activation of $\beta$-catenin and Akt pathways by Twist are critical for the maintenance of EMT associated cancer stem cell-like characters. BMC Cancer 11: 49, 2011.

22. Zhuo WL, Wang Y, Zhuo XL, Zhang YS and Chen ZT: Short interfering RNA directed against TWIST, a novel zinc finger transcription factor, increases A549 cell sensitivity to cisplatin via MAPK/mitochondrial pathway. Biochem Biophys Res Commun 369: 1098-1102, 2008.
23. Sakowicz-Burkiewicz M, Przybyla T, Wesserling $M$ Bielarczyk H, Maciejewska I and Pawelczyk T: Suppression of TWIST1 enhances the sensitivity of colon cancer cells to 5-fluorouracil. Int J Biochem Cell Biol 78: 268-278, 2016.

24. Stavrovskaya AA and Stromskaya TP: Transport proteins of the ABC family and multidrug resistance of tumor cells. Biochemistry (Mosc) 73: 592-604, 2008.

25. Fletcher JI, Haber M, Henderson MJ and Norris MD: ABC transporters in cancer: More than just drug efflux pumps. Nat Rev Cancer 10: 147-156, 2010.

26. Johnatty SE, Beesley J, Paul J, Fereday S, Spurdle AB, Webb PM, Byth K, Marsh S, McLeod H, Harnett PR, et al; AOCS Study Group: ABCB1 (MDR 1) polymorphisms and progression-free survival among women with ovarian cancer following paclitaxel/ carboplatin chemotherapy. Clin Cancer Res 14: 5594-5601, 2008.

27. $\mathrm{Xu} \mathrm{D,} \mathrm{Lu} \mathrm{Q} \mathrm{and} \mathrm{Hu} \mathrm{X}$ : Down-regulation of P-glycoprotein expression in MDR breast cancer cell MCF-7/ADR by honokiol. Cancer Lett 243: 274-280, 2006.

28. Zhu K, Chen L, Han X, Wang J and Wang J: Short hairpin RNA targeting Twist1 suppresses cell proliferation and improves chemosensitivity to cisplatin in HeLa human cervical cancer cells. Oncol Rep 27: 1027-1034, 2012.

29. Lu S, Yu L, Mu Y, Ma J, Tian J, Xu W and Wang H: Role and mechanism of Twist1 in modulating the chemosensitivity of FaDu cells. Mol Med Rep 10: 53-60, 2014.

30. Shen W, Pang H, Liu J, Zhou J, Zhang F, Liu L, Ma N, Zhang N, Zhang $\mathrm{H}$ and Liu L: Epithelial-mesenchymal transition contributes to docetaxel resistance in human non-small cell lung cancer. Oncol Res 22: 47-55, 2014.

31. Biddle A and Mackenzie IC: Cancer stem cells and EMT in carcinoma. Cancer Metastasis Rev 31: 285-293, 2012.

32. Ishiwata T: Cancer stem cells and epithelial-mesenchymal transition: Novel therapeutic targets for cancer. Pathol Int 66: 601-608, 2016.

33. Liang L, Sun H, Zhang W, Zhang M, Yang X, Kuang R and Zheng H: Meta-analysis of EMT datasets reveals different types of EMT. PLoS One 11: e0156839, 2016.

34. Carnero A, Garcia-Mayea Y, Mir C, Lorente J, Rubio IT and LLeonart ME: The cancer stem-cell signaling network and resistance to therapy. Cancer Treat Rev 49: 25-36, 2016.

35. Zhu Z, Hao X, Yan M, Yao M, Ge C, Gu J and Li J: Cancer stem/progenitor cells are highly enriched in $\mathrm{CD} 133^{+} \mathrm{CD} 44^{+}$ population in hepatocellular carcinoma. Int J Cancer 126: 20672078, 2010

36. Sahlberg SH, Spiegelberg D, Glimelius B, Stenerlöw B and Nestor M: Evaluation of cancer stem cell markers CD133, CD44, CD24: Association with AKT isoforms and radiation resistance in colon cancer cells. PLoS One 9: e94621, 2014.

37. Horst D, Kriegl L, Engel J, Kirchner T and Jung A: Prognostic significance of the cancer stem cell markers CD133, CD44, and CD166 in colorectal cancer. Cancer Invest 27: 844-850, 2009.

38. Elander N, Söderkvist P and Fransén K: Matrix metalloproteinase (MMP) $-1,-2,-3$ and -9 promoter polymorphisms in colorectal cancer. Anticancer Res 26 (1B): 791-795, 2006.

39. Roomi MW, Monterrey JC, Kalinovsky T, Rath M and Niedzwiecki A: Patterns of MMP-2 and MMP-9 expression in human cancer cell lines. Oncol Rep 21: 1323-1333, 2009. 